\title{
Adaptive Beam Forming Using KLMS, KRLS and CGM Algorithms
}

\author{
G. KranthiKumar ${ }^{1}$, Dr. A. Jhansi Rani ${ }^{2}$, Ch.Raghavendra ${ }^{3}$, M. Sunitha ${ }^{4}$ \\ 1, 2, 3,4 (Department of ECE, Velagapudi Ramakrishna Siddhartha Engineering College, Vijayawada, INDIA)
}

\begin{abstract}
In this paper Adaptive beam forming using KLMS, CGM and KRLS algorithm has been proposed. In CGM algorithms has reduced the interference and convergence is better when number of antennas increased. In CGM algorithm the weight of antenna arrays can be adjusted to form certain amount of adaptive beam to track corresponding users automatically and at the same time to minimize interference arising from other users by introducing nulls in their directions. The experimental results show that the different algorithms of array factors, mean square error and performance of different antennas on each algorithm. The CGM algorithm has better convergence, low computational complexity and minimizing interference compare to other algorithms.
\end{abstract}

Keywords: Antenna Arrays, Adaptive Algorithms, Beam-forming, Interference, Smart antenna, Signal Nulling.

\section{INTRODUCTION}

Smart antenna for mobile communication has received enormous interests worldwide in recent years. In the last decade wireless cellular communication has experienced rapid growth in the demand for provision of new wireless multimedia services such as internet access, multimedia data transfer and video conferencing. In order to meet this demand and to overcome the limited capacity of conventional single input single output (SISO) systems, the use of multiple element antennas (MEAs) has been under consideration. A multiple input multiple output (MIMO) system offers greater capacity than SISO counterparts [1]. Smart antenna techniques, such as MIMO systems, can extend the capabilities of $3 \mathrm{G}$ and $4 \mathrm{G}$ systems to provide customers with increased data throughput for mobile high-speed data applications. MIMO systems use multiple antennas at both the transmitter and receiver to increase the capacity of the wireless channel. With these techniques, it may be possible to provide in excess of $1 \mathrm{Mbit} / \mathrm{sec}$ for the $3 \mathrm{G}$ wireless systems and $20 \mathrm{Mbits} / \mathrm{sec}$ for $4 \mathrm{G}$ systems.

In $4 \mathrm{G}$ wireless system, cellular providers have the opportunity to offer data access to a wide variety of devices. The cellular network would become a data network on which cellular phones could operate as well as any other data device. Sending data over the cell phone network is a profitable business. Wireless networks provide a unique twist to this product: mobility. This concept is already beginning a revolution in wireless networking, with instant access to the Internet from anywhere.

A smart antenna consists of array antenna, whose signals are processed adaptively in order to exploit the spatial dimension of the mobile radio channel. All elements of the antenna array have to be weighted in order to adapt to the current channel and desired user characteristics. This weight adaptation is the "smart" part of the smart antenna; hence it called as "adaptive antenna". The term "smart" implies the use of signal processing in order to shape the beam pattern according to certain conditions. In smart antenna system, desired signals can be distorted by interfering signal that arrives at the antenna of the receiver. If the interfering signal is arriving at an angle different from the desired signal, the interference could be reduced in the direction of the desired signal.

\section{Smart Antennas}

Smart antennas are antenna arrays with smart signal processing algorithms used to identify spatial signal signature such as the direction of arrival (DOA) of the signal, and use it to calculate beamforming vectors, to track and locate the antenna beam on the mobile/target. These can provide higher system capacities, increase signal to noise ratio, higher bit rate, higher Coverage, Improved link quality, spectral efficiency, Mobility, reduce interference, reduce multipath and co-channel interference by steering the main beam towards the user and at the same time forming nulls in the directions of the interfering signal [2].

There are two different types of smart antennas:

\section{Switched Beam}

Switched beam systems have several available fixed beam patterns. A decision is made as to which beam to access, at any given point in time, based upon the requirements of the system. 
2. Adaptive array

Adaptive arrays allow the antenna to steer the beam to any direction of interest while simultaneously null interfering signals.

\section{A. Theoretical model for smart antennas system}

The smart antennas generally refer as antenna array is combined with digital signal processing to give a narrow beam pattern in response to the received signal by the smart antenna. The weight of the beamformer is calculated by using the adaptive approach which uses reference signal and the direction of the user based on the received signal. The main beam is then directed toward the specific user, while the nulls are adjusted toward the interferers. Similarly, there are many methods and algorithms to update the array weights, each with its speed of convergence and required processing time [3].

The smart antenna system can be divided mainly into three parts.

1. The first one performs the direction of arrival (DOA) estimation and determines the received input data in uplink the number of incoming wave fronts.

2. The second part performs the DOA classification. It finds which wave fronts originate from the user and which ones from the interferers.

3. The third part is the beam forming algorithm. It forms an antenna pattern with a main beam steered in the direction of the user, while minimizing the influence of the interfering wave fronts and the noise. Fig 1 shows the functional block diagram of smart antenna system.

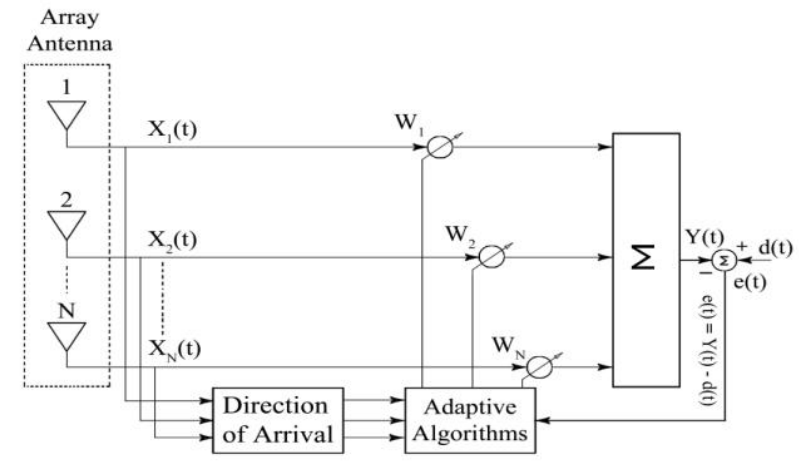

Fig 1. Functional Block Diagram of Smart Antennas

\section{Different Beamforming Algorithm}

Adaptive beam forming is a technique in which an array of antennas is exploited to achieve maximum reception in a specified direction by estimating the desired direction while signals of the same frequency from other directions are rejected. This process is achieved by varying the weights of each of the sensors used in the array. This allows the antenna system to focus the maxima of the antenna pattern towards the desired mobile while minimizing the impact of noise, interference and other effects from undesired mobiles [4].

The present research paper describes about simulated adaptive beam former (KLMS), (KRLS) algorithm and Conjugate Gradient Method (CGM). The output of the array is the weighted sum of the received signals at the array elements and the noise at the receivers connected to each element. The weights iteratively computed based on the array output a reference signal that approximates the desired signal and previous weights. The reference signal is approximated to the desired signal. An adaptive processor will minimize the between a desired signal and array output [4].

\section{A. KLMS TECHNIQUE}

The LMS Algorithm is the most widely used adaptive beamforming algorithm, being employed in several communication application. The LMS Algorithm changes the weight vector along the direction of the estimated gradient based on the steepest descent method [3].The weight vector updating for LMS Algorithm is given by

$$
\begin{aligned}
& w(n+1)=w(n)+\mu x^{*}(n) e(n) \\
& e(n)=d(n)-X(n) w(n)
\end{aligned}
$$

The Kalman filter is an algorithm which uses a series of measurements observed over time, containing noise (random variations) and other inaccuracies, and produces estimates of unknown variables that tend to be more precise than those that would be based on a single measurement alone. The Kalman filter operates recursively on streams of noisy input data to produce a statistically optimal estimate of the underlying system 
state [5]. The kalman based LMS algorithm is less sensitive to the measurement noise. It also ensures fast convergence, high accuracy in the weight estimate, low misadjustment, stability and decreased computational complexity.

The algorithm works in a two-step process: in the prediction step, the Kalman filter produces estimates of the current state variables, along with their uncertainties. Once the outcome of the next measurement is observed, these estimates are updated using a weighted average, with more weight being given to estimates with higher certainty. Because of the algorithm's recursive nature, it can run in real time using only the present input measurements and the previously calculated state [5].

The weight update equation of the kalman based normalised Least Mean Square (KLMS):

$$
\begin{gathered}
w(n+1)=w(n)+\frac{x(n) e(n)}{S(n)+\frac{q v}{\sigma w(n)}} \\
\sigma w(n+1)=\sigma w(n)\left(1-\frac{\frac{S(n)}{(N-1)}}{S(n)+\frac{q v}{\sigma w(n)}}\right.
\end{gathered}
$$

$S(n)=x^{T}(n) x(n)$

The state noise and measurement noise of the kalman filter state model is assumed to be Gaussian random variables with known autocorrelation functions.

\section{B. KRLS TECHNIQUE}

The convergence speed of the LMS algorithm depends on the eigen values of the array correlation matrix. In an environment yielding an array correlation matrix with large eigen value spread the algorithm converges with a slow speed. The RLS requires reference signal and correlation matrix information. An important feature of the recursive least square algorithm is that its convergence rate is faster than the LMS algorithm. This problem is solved with the RLS algorithm by replacing the gradient step size $\mu$ with a gain matrix $R^{-1}(n)$ at the $\mathrm{n}^{\text {th }}$ iteration, producing the weight update equation.

The weight vector updating for RLS algorithm is given by

$$
w(n)=w(n-1)-R^{-1}(n) \xi(n)
$$

Where the error is given by

$$
\begin{aligned}
& \xi(n)=d(n)-X^{T}(n) w^{*}(n-1) \\
& R^{-1}(n)=\frac{1}{\delta_{0}}\left[\Lambda^{\Lambda^{-1}} R(n-1)-\frac{R(n-1) x(n) x^{H}(n) R(n-1)}{\delta_{0}+x^{H}(n) R(n-1) x(n)}\right]
\end{aligned}
$$

Kalman based normalised Recursive Least Square (KRLS) algorithm is an adaptive beam forming algorithm. The optimal weight value is obtained by means of iterative operation according to the rule of MMSE (minimum mean square error). Adaptive algorithm computes the output based on received signal and weight vector. The weight vector is adjusted based on the error between output and expected signal. The above progress is continuing iterative operation course until it satisfies the demand and comes into the steady state.

The weight updates equation of the Kalman based normalised RLS [3] is given below,

According to Error Vector $\xi(n)$ eq (7), weights update $w(n-1)$ eq (3), $\sigma w(n)$ eq (4) and $S(n)$ eq (5). Calculated as:

$$
w(n)=w(n-1)+\frac{R^{-1}(n) \xi(n)}{S(n)+\frac{q v}{\sigma w(n)}}
$$

$q v$ is the autocorrelation function of the state noise. 


\section{CGM TECHNIQUE}

The problem with the steepest descent method has been the sensitivity of the convergence rates of the eigen value spread of the correlation matrix. Greater spreads result in slower convergences. The convergence rate can be accelerated by use of the conjugate gradient method (CGM). The goal of CGM is to iteratively search for the optimum solution by choosing conjugate (perpendicular) paths for each new iteration. The method of CGM produces orthogonal search directions resulting in the fastest convergence. The path taken at iteration $(n-1)$ is perpendicular to the path taken at the previous iteration $n$ [2].

Conjugate gradient method is generally useful for solving a set of equations of the form $(A w=d)$ to obtain the value of $w$. In fact, CGM produces weights vector, at each sample of time, corresponding to the complex conjugate of the optimum weights vector of the smart antenna system. Therefore, it is suitable to modify the equation to $\left(A w^{*}=d\right)$ where $*$ denotes the complex conjugate [2].

Consider $A$ represents $K \times M$ matrix of array snapshots, $K$ denotes a specific number of iteration, $d$ represents the reference signal (assumed to be similar to the desired signal) of $K$ snapshots. In other word, $A$ and $d$ can be given by [3].

$$
\begin{aligned}
A & =\left[\begin{array}{ccccc}
x_{1}(1) & x_{2}(1) & x_{3}(1) & \cdots & x_{M}(1) \\
x_{1}(2) & x_{2}(2) & x_{3}(2) & \cdots & x_{M}(2) \\
x_{1}(3) & x_{2}(3) & x_{3}(3) & \cdots & x_{M}(3) \\
\vdots & \vdots & \vdots & \ddots & \vdots \\
x_{1}(K) & x_{2}(K) & x_{3}(K) & \cdots & x_{M}(K)
\end{array}\right]_{K \times M} \\
d & =\left[\begin{array}{c}
S_{0}(1) \\
S_{0}(2) \\
S_{0}(3) \\
\vdots \\
S_{0}(K)
\end{array}\right]
\end{aligned}
$$

The residual vector $(r)$ denotes the error between the reference signal and the array output signal at each iteration. Thus, $r$ can be expressed as [7].

$r=d-A w^{*}$

CGM starts with an initial guess of the weights vector then obtain the residual $\left(r(0)=d-A w^{*}(0)\right)$. Subsequently the direction vector $(D)$ which gives the new conjugate direction toward the optimum weights is given by [3].

$$
D=A^{H} r
$$

To update the weights vector, the following expression can be used [2].

$$
w^{*}(n+1)=w^{*}(n)-\mu(n) D(n)
$$

Where $\mu(n)$ represents the step size at $n^{\text {th }}$ iteration which can be expressed as [2].

$$
\mu(n)=\frac{r^{H}(n) A A^{H} r(n)}{D^{H}(n) A A^{H} r(n)}
$$

After that, $r$ and $D$ should be updated using the following expressions [3].

$$
\begin{aligned}
& r(n+1)=r(n)+\mu(n) D(n) \\
& D(n+1)=A^{H} r(n+1)-\alpha(n) D(n)
\end{aligned}
$$

and the value of $\alpha(n)$ that minimizes the error function can be given by [3].

$$
\alpha(n)=\frac{r^{H}(n+1) A A^{H} r(n+1)}{r^{H}(n) A A^{H} r(n)}
$$

When the updating process is over, a new step size and new weights vector are generated using (15) and (14) respectively and so on. It can be shown that true solution can be found in no of more than iteration. This condition is known as quadratic convergence [7]. 
IV. Results And Discussion

MATLAB is used to perform simulations of the adaptive algorithms discussed are in section 3 . The simulated algorithms are given below.

A. Analysis of Kalman Based Normalized Least Mean Square (KLMS) algorithm.

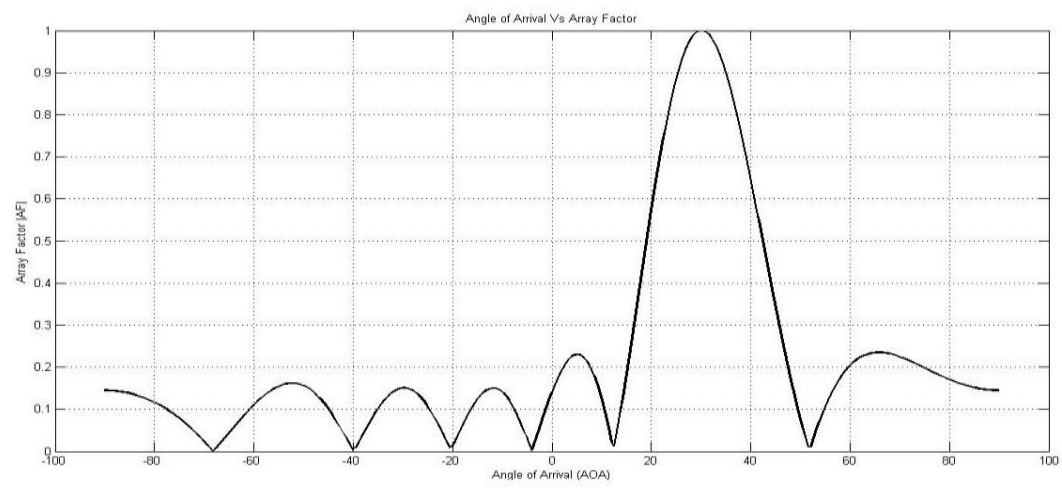

Fig 2. Angle of Arrival Vs Array Factor: Beam steered at $30^{\circ}$ (Signal of interest) and interferer null at $-20^{\circ}$ using KLMS algorithm.

It is considered that the desired user is arriving at an angle $30^{\circ}$ and an interferer at an angle $-20^{\circ}$. The array factor is computed for 8 elements and the Fig 2 shows the array factor plots and how the KLMS algorithm places deep nulls in the direction of interfering signals and maximum in the direction of the desired signal.

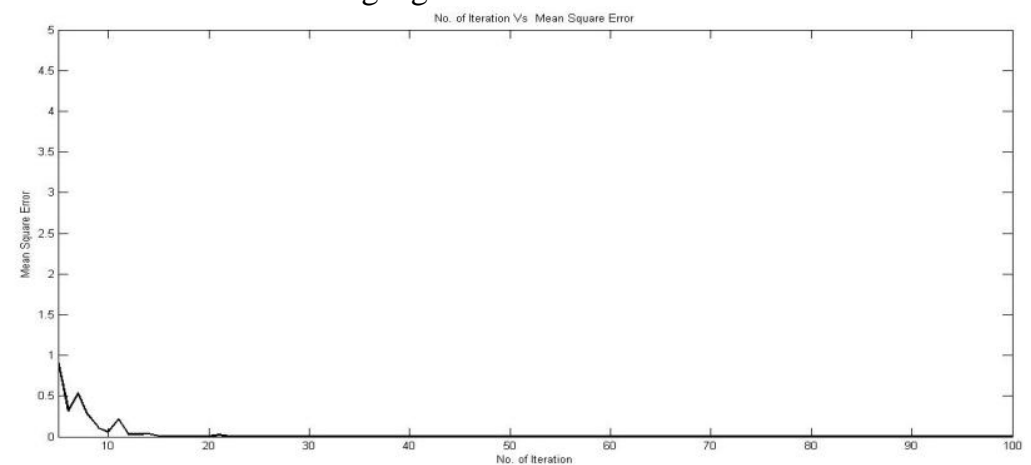

Fig 3. No. of Iterations Vs Mean square error, the desired signal and the array output signal at each iteration which converges toward zero after 13 iteration

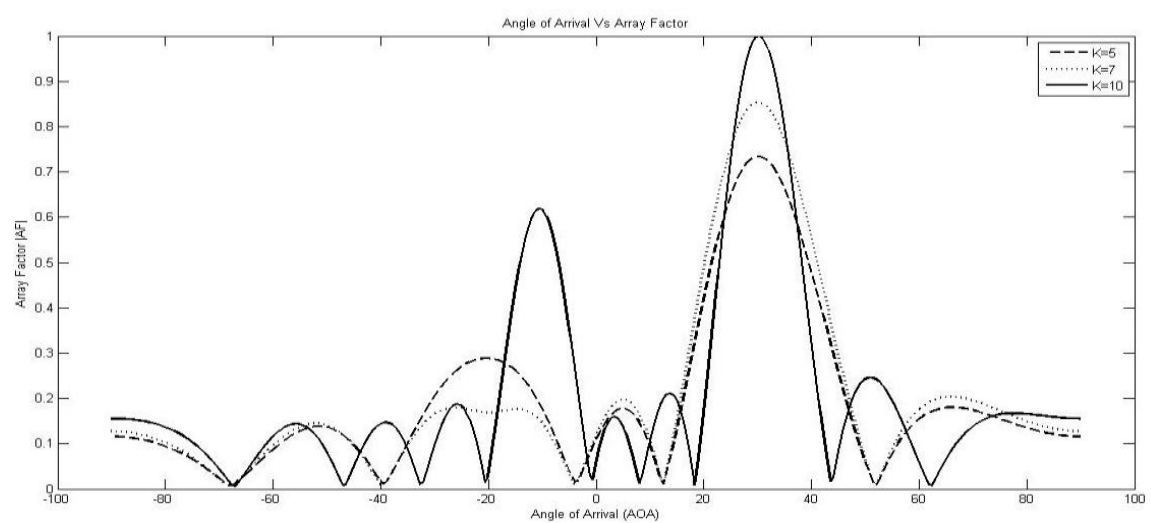

Fig 4. Comparison the Normalize array factor of KLMS. Angle of arrival Vs array factor, Beam steered at $30^{\circ}$ (Signal of interest) and interferer nulled at $-\mathbf{7 0}^{\circ}$.

The Figure 4 shows KLMS algorithm places adaptively the maxima in the direction of desired user and nulls at the AOA of the interferer for various values of $\mathrm{N}$. Simulation results proved the higher Value of antenna element gives better results.

B. Analysis of Kalman Based Normalized Recursive Least Square (KRLS) algorithm 


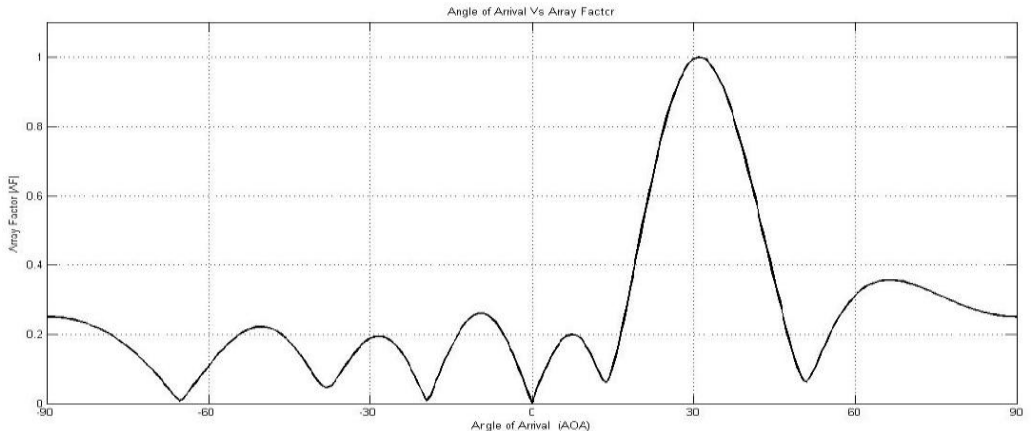

Fig 5. Angle of Arrival Vs Array Factor: Beam steered at $30^{\circ}$ (Signal of interest) and interferer nulled at $0^{\circ}$ using KRLS algorithm.

It is considered that the desired user is arriving at an angle $30^{\circ}$ and an interferer at an angle $0^{0}$. The array factor is computed for 8 elements and the Fig 5 shows the array factor plots and how the KRLS algorithm places deep nulls in the direction of interfering signals and maximum in the direction of the desired signal.

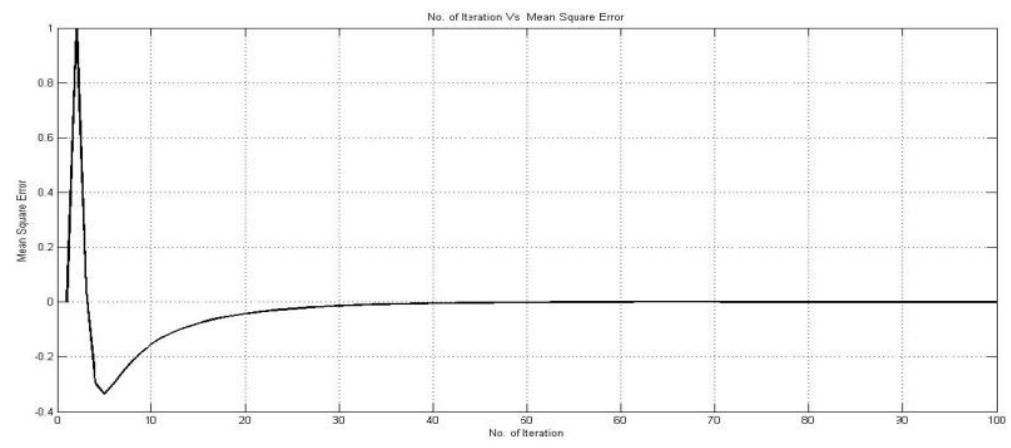

Fig 6. No. of Iterations Vs Mean square error, the desired signal and the array output signal at each iteration which converges toward zero after 25 iteration

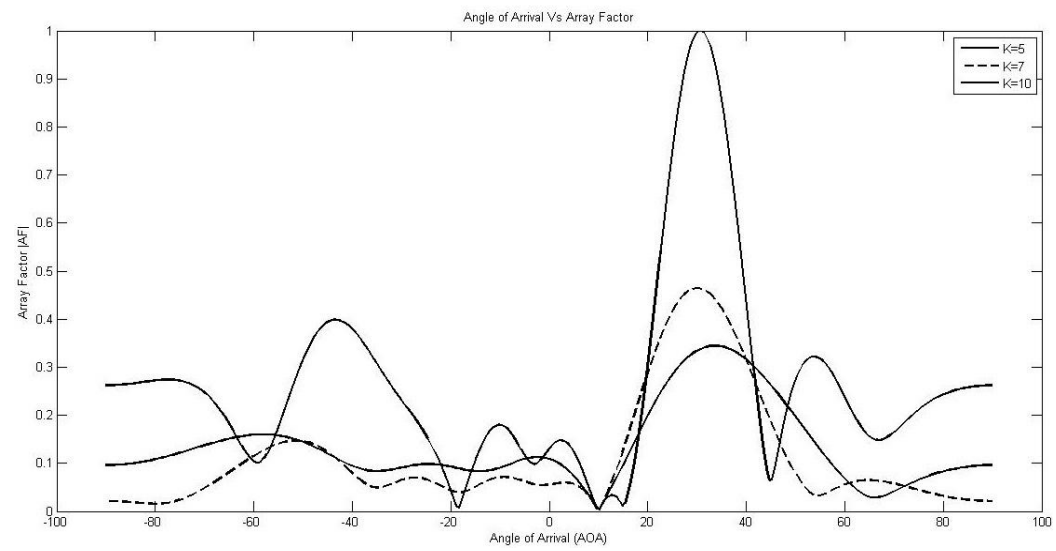

Fig 7. Comparison the Normalize array factor of KRLS. Angle of arrival Vs array factor, Beam steered at $30^{\circ}$ (Signal of interest) and interferer nulled at $10^{\circ}$.

The Fig 7 shows KRLS algorithm places adaptively the maxima in the direction of desired user and nulls at the AOA of the interferer for various values of N. Simulation results prove that higher Value of antenna element gives better results.

In Fig 6 and Fig 3 shows the error response of KRLS and KLMS algorithm for N=8. Error response of KRLS is low compare to KLMS. For example amplitude of KRLS at $10^{\text {th }}$ sample is negative value; it is less than KLMS as shown in Fig 6 and Fig 3.

\section{Analysis of Conjugate Gradient Method (CGM)}




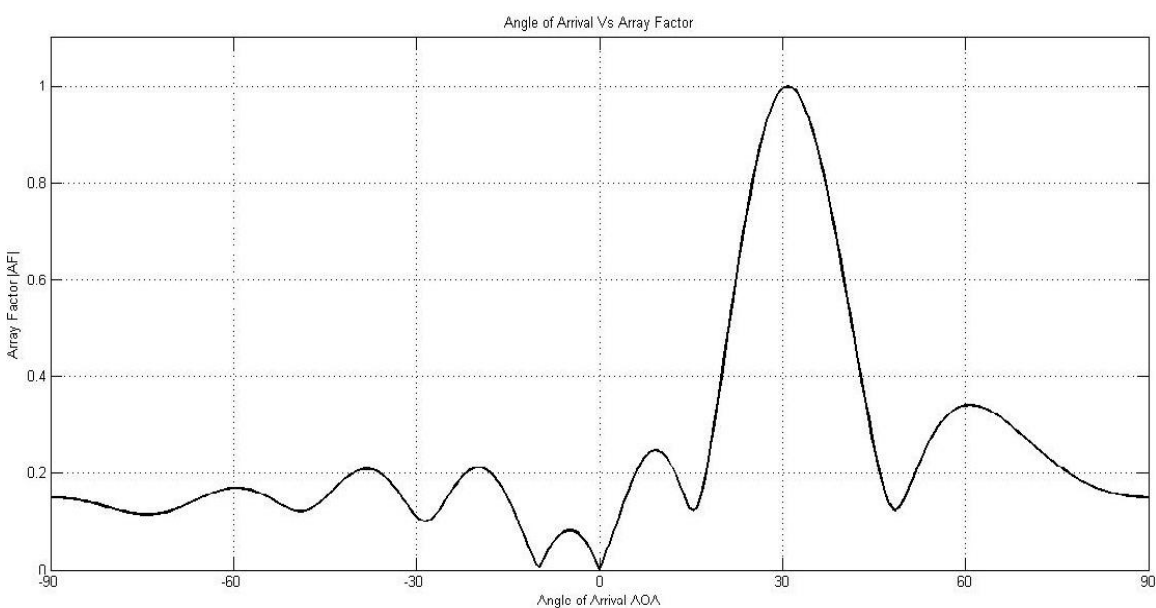

Fig 8. Angle of Arrival Vs Array Factor: Beam steered at $30^{\circ}$ (Signal of interest) and interferer nulled at $0^{\circ}$ and -10 using CGM algorithm.

It is considered that the desired user is arriving at an angle $30^{\circ}$ and an interferer at an angle $0^{\circ}$ and $-10^{\circ}$. The array factor is computed for 8 elements and the Figure 8 shows the array factor plots and how the KLMS algorithm places deep nulls in the direction of interfering signals and maximum in the direction of the desired signal.

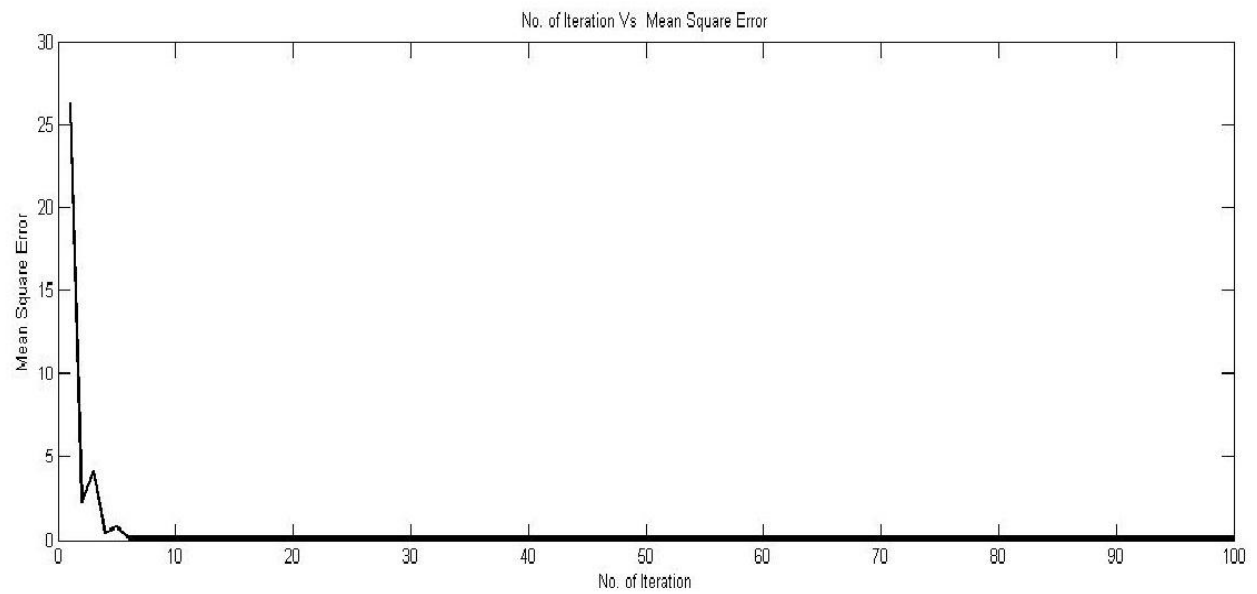

Fig 9. No. of Iterations Vs Mean square error, the desired signal and the array output signal at each iteration which converges toward zero after 5 iteration

Fig 9. Illustrates the mean square error (MSE) between the desired signal and the array output signal at each iteration which converges toward zero after five iteration. In Fig 6, Fig 3 and Fig 9 shows the error response of KRLS, KLMS and CGM algorithm for $\mathrm{N}=8$.Error response of CGM is low compare to KLMS, KRLS.

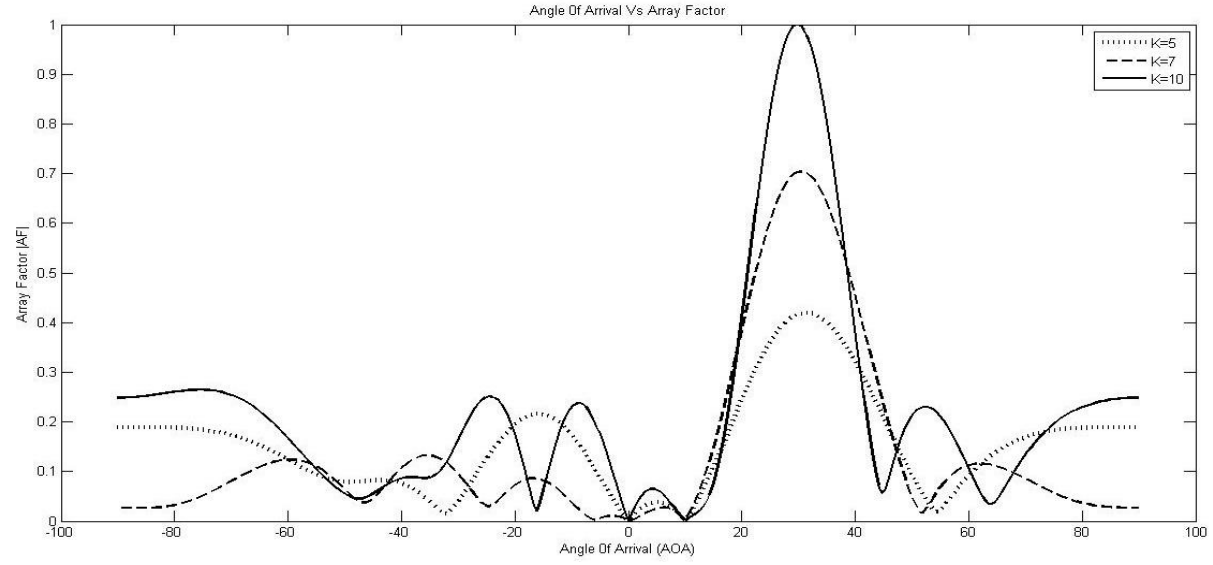


Fig 10. Comparison the Normalize array factor of CGM. Angle of Arrival Vs Array Factor, Beam steered at $30^{\circ}$ and interferer nulled at $10^{\circ}$ and $0^{\circ}$.

The Fig 10 shows CGM algorithm places adaptively the maxima in the direction of desired user and nulls at the AOA of the interferer for various values of N. Simulation results prove that higher Value of antenna element gives better results.

\section{Comparison the Normalize array factor using different algorithms}

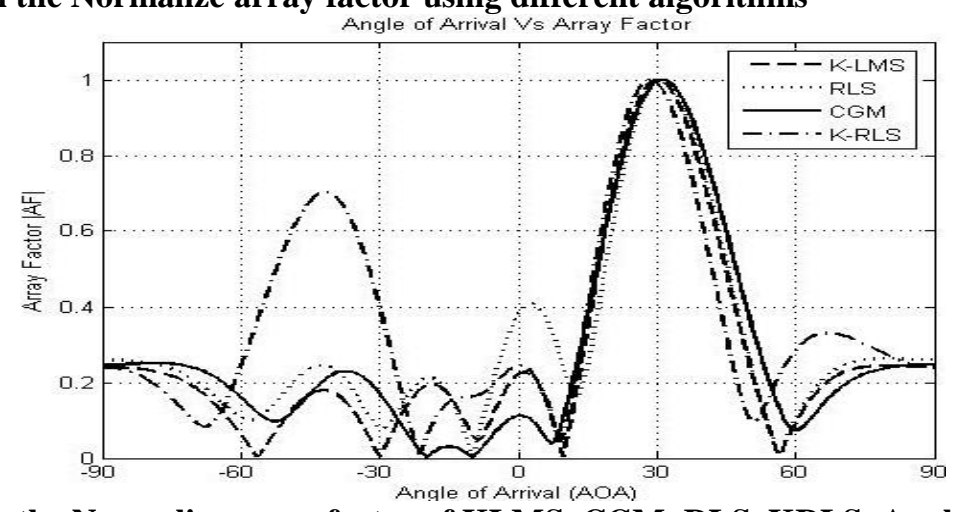

Fig 11. Comparison the Normalize array factor of KLMS, CGM, RLS, KRLS. Angle 0f Arrival Vs Array Factor, Beam steered at $30^{\circ}$ (Signal of interest), interferer nulled at $10^{\circ}$ and No. of Elements $\mathrm{N}=5$.

The Fig 11 shows different algorithms places adaptively the maxima in the direction of desired user and nulls at the AOA of the interferer.

\section{CONCLUSION}

In this paper, adaptive beam forming using KLMS, KRLS and CGM has been proposed. CGM has better convergence compare to KLMS and KRLS technique. The computational complexity of CGM is low and minimizing interference from other users by introducing nulls in their directions. The results shows that when the arrival angel of expected users' signal coming within certain range of angel of incidence, pattern can clearly differentiate the expected signal and the interference signal, and form notch for the interference signal, that is to say, smart antenna with different structures correspond relevant direction and range.

\section{References}

[1] S.F. Shaukat, Mukhtarul Hassan, R. Farooq, H.U. Saeed and Z. Saleem, "Sequential Studies Beamforming Algorithms for smart Antenna Systems", World Applied Sciences Journal Vol. 6, pp.754-758, ISSN 1818-4952, 2009.

[2] Frank Gross, "Smart Antenna for Wireless Communication", McGraw-hill, September 14, 2005.

[3] AnuragShivam Prasad, SandeepVasudevan, Selvalakshmi R, Sree Ram K, Subhashini G, Sujitha S, "Analysis of Adaptive Algorithms for Digital Beamforming in Smart Antennas", IEEE-International Conference on Recent Trends in Information Technology (ICRTIT), pp. 64-68, ISSN 978-1-4577-0590- 8/11/\$26.00, 2011 IEEE.

[4] Ch. Santhi Rani, P. V. Subbaiah, K. Chennakesava Reddy and S. Sudha Rani, "LMS and RLS Algorithms for Smart Antennas in a W-CDMA Mobile Communication Environment", Asian Research Publishing Network (ARPN) Journal of Engineering and Applied Sciences, Vol. 4, pp. 78-88, ISSN 1819-6608, August 2009.

[5] SymonHaykin, "Adaptive filter theory”, Forth edition, Pearson Education, Asia, 2002.

[6] KhyatiR.Zalawadia, Twinkle V. Doshi ,Dr U D Dalal, "Adaptive Beam Former Design using RLS Algorithm for Smart Antenna System", International Conference on Computational Intelligence and Communication Systems,pp. 102-106, 2011.

[7] Lal Chand Godara, "Smart Antennas", CRC Press LLC, 2004.

[8] Constantine A.Balanis, “Antenna-Theory, Analysis and Design”, Edition 2, JOHN WILEY \& SONS,INC, 2009.

[9] Frank B. Gross, "Smart Antennas for Wireless Communications with MATLAB", McGraw-hill, 2005.

[10] Mousa, F.M., "Weights vector adaptation of smart antenna system using conjugate gradient method" International Conference Next Generation Information Technology (ICNIT), pp. 6-9, 2011. 